\title{
Cytologic Findings of Breast Mucinous Carcinoma with Micropapillary Pattern: Report of A Case and Literature Review
}

\section{Chih-Yi Liu' ${ }^{1 *}$, Chiang-Shin Liu ${ }^{2}$ and Yih-Yiing Wu ${ }^{1}$}

${ }^{1}$ Department of Pathology, Cathay General Hospital, Sijhih, Taipei, Taiwan

${ }^{2}$ Department of Pathology, Cathay General Hospital, Taipei, Taiwan

\begin{abstract}
We present a case of breast mucinous carcinoma with micropapillary pattern, which shows cytologic features equivalent to those seen in both mucinous carcinoma and micropapillary carcinoma. Subsequent histology further confirms the diagnosis. It is possible that this peculiar morphologic subtype and its clinical significance have been under-recognized.
\end{abstract}

Keywords: Mucinous carcinoma; Breast; Micropapillary carcinoma; Fine-needle aspiration cytology

\section{Introduction}

Mucinous lesions of the breast represent a broad spectrum of entities, which may pose a diagnostic challenge on fine-needle aspiration cytology. Mucinous carcinomas are so bland cytologically that they may be misdiagnosed as benign lesions, especially in cellpoor samples with ample mucin [1]. Assessment of cytologic details in conjunction with the radiological and clinical findings leads to a more accurate diagnosis. In addition, micropapillary carcinoma of the breast is known for the high risk of lymphatic invasion and metastasis. Wai-Kuen $\mathrm{Ng}$ has suggested a new subtype of mucinous carcinoma, morphologically equivalent to a mucinous counterpart of micropapillary carcinoma, in a large review series [2]. A recent study by Ranade et al. [3] has described that pure mucinous breast carcinoma with a micropapillary pattern were more frequently associated with nodal disease [3]. Thus prompt axillary staging is recommended in these patients. Herein we present a case of mucinous carcinoma with micropapillary pattern, demonstrating the cytologic and histologic features that warrant special attention.

\section{Case Report}

An 83-year-old Taiwanese woman complained of left breast mass for two months. Her past medical record and family history were unremarkable. Sonography revealed a partially ill-defined and lobulated tumor about $2.6 \times 1.2 \mathrm{~cm}$ in dimension (Figure 1). Fineneedle aspiration of the breast tumor was also performed.

The fine-needle aspiration smear was moderately cellular with clusters of tumor cells against a rich mucinous background (Figure 2a). The tumor cells were bathed in wispy or colloid-like mucin material. Tightly cohesive three-dimensional cell balls and angulated clusters were noticed. There were few branching fragments and abortive micropapillae, as well as dispersed single epithelial cells (Figure 2b). The tumor cells exhibited mild to moderate nuclear atypia with small nucleoli and scattered intracytoplasmic vacuoles. Occasionally, thinwalled capillaries transversing the mucinous material were observed. Oval bare nuclei, fragments of fibromyxoid stroma and necrotic debris were not present in the smear. Accordingly, the cytologic diagnosis was ductal carcinoma with mucinous features.

The patient received a subsequent excisional biopsy. The excised tumor was composed of neoplastic cells growing in micropapillae, pseudoacini, trabeculae and focal cribriform pattern, and floating in the mucinous pools (Figure 3a). The tumor border was relatively well-circumscribed, with focal presence of irregular infiltration. The micropapillary architecture was formed by clusters of tumor cells with a central space and a serrated outer border (Figure 3b). Mild nuclear pleomorphism with distinct nucleoli was seen. Focal nuclear hobnailing was also noticed. The above picture led to the diagnosis of mucinous carcinoma with micropapillary pattern.

Two weeks later, modified radical mastectomy with axillary lymph node dissection was performed. Evident vascular space permeation and nodal metastasis were not present in the surgical specimen. In immunohistochemical study, approximately $70 \%$ of the tumor cells were weakly to moderately positive for estrogen receptor (ER). Reactivity for progesterone receptor (PR) was not identified. The neoplastic cells showed diffuse and strong membranous staining for HER-2/neu protein (score: $3+$ ).

\section{Discussion}

Breast lesions with mucin represent a broad spectrum of entities, including fibrocystic change (FCC) with luminal mucin, mucocelelike lesion (MLL), pure or mixed type of mucinous carcinoma, and other conditions accompanied by mucin-like material. Among theses mucinous lesions, MLL is an uncommon tumor initially described by Rosen as a benign process of breast [4]. The subsequent reports on MLLs disclose a spectrum of pathologic lesions from benign tumor, atypical ductal hyperplasia, carcinoma in situ to invasive carcinoma, further complicating the diagnostic problem. Mucinous carcinoma is a variant of breast cancer, characterized by the accumulation of abundant extracellular mucin around invasive carcinoma cells. In practice, a carcinoma should not be classified as pure mucinous carcinoma if more than $10 \%$ of the invasive component is nonmucinous, or if the nonmucinous invasive component is poorly differentiated cytologically [4]. In general, pure mucinous carcinomas have a favorable prognosis, and the ten-year survival ranges from $80 \%$ to $100 \%$.

*Corresponding author: Chih-Yi Liu M.D, Department of Pathology, Cathay General Hospital, Sijhih, Taipei, Taiwan, Tel: 886-2-26482121 ext.3741; Fax: 8862-82370777; E-mail: cyl1124@gmail.com

Received February 18, 2011; Accepted March 09, 2011; Published March 11 2011

Citation: Liu CY, Liu CS, Wu YY (2011) Cytologic Findings of Breast Mucinous Carcinoma with Micropapillary Pattern: Report of A Case and Literature Review. J Cytol Histol 2:114. doi:10.4172/2157-7099.1000114

Copyright: @ 2011 Liu CY, et al. This is an open-access article distributed unde the terms of the Creative Commons Attribution License, which permits unrestricted use, distribution, and reproduction in any medium, provided the original author and source are credited. 
Citation: Liu CY, Liu CS, Wu YY (2011) Cytologic Findings of Breast Mucinous Carcinoma with Micropapillary Pattern: Report of A Case and Literature Review. J Cytol Histol 2:114. doi:10.4172/2157-7099.1000114

The cytologic features of mucinous carcinoma are well established. However, aspirates with abundant extracellular mucinous material originating from other mammary lesions, especially those with increased cellularity, may pose a diagnostic challenge on fine needle aspiration

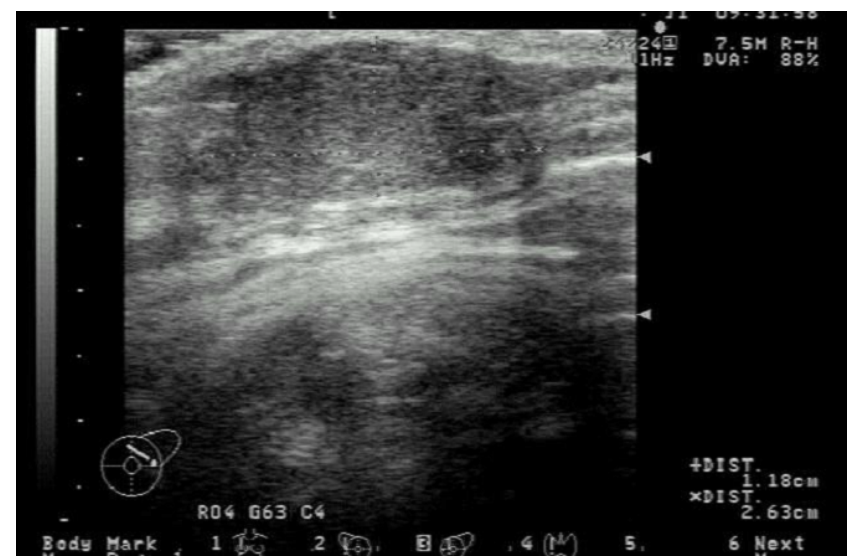

Figure 1: Sonography revealed a lobulated tumor mass with partially ill-defined border.
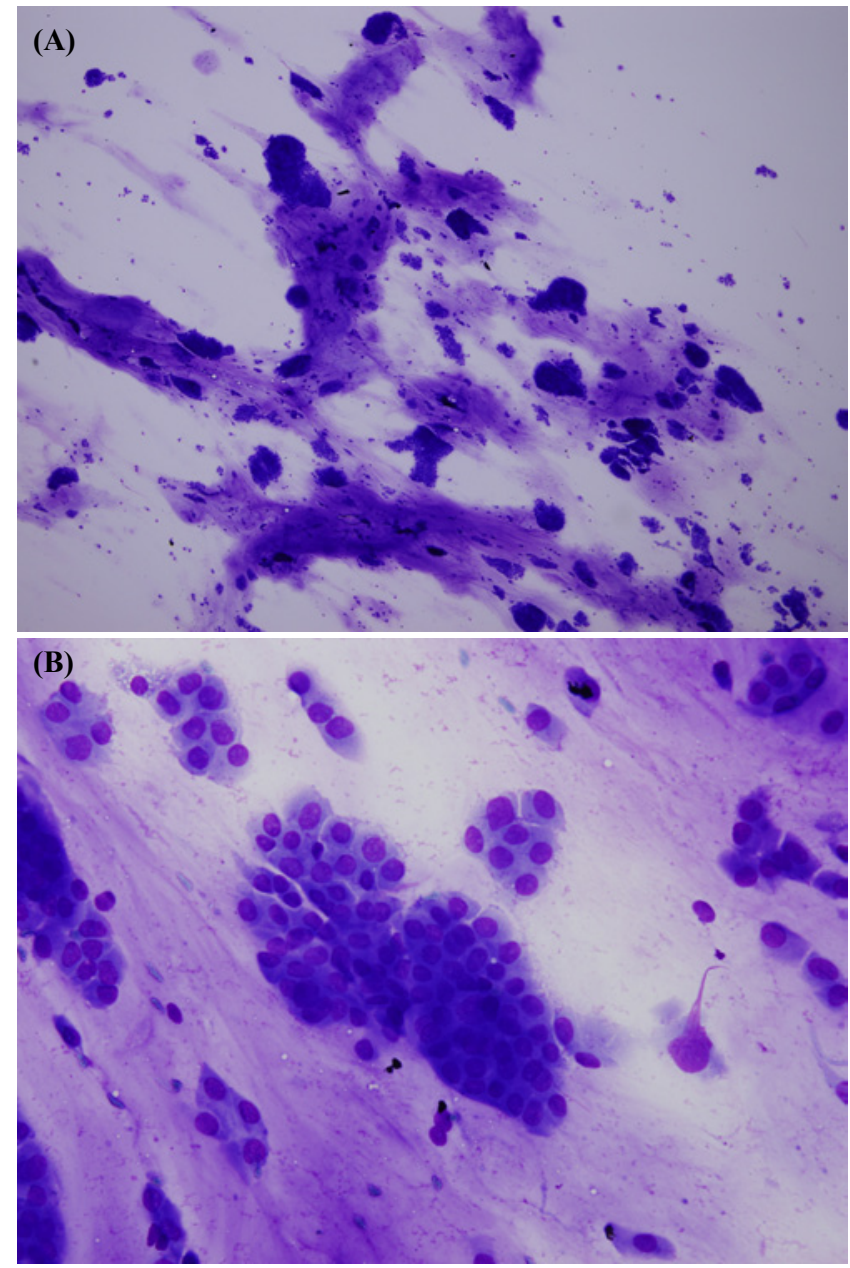

Figure 2: Fine-needle aspiration smear showed clusters of tumor cells in a rich mucinous background (a). Abortive micropapillae, trabeculae or dispersed single tumor cells were observed (b). (Liu stain; $a, x 40 ; b, x 200$ ).
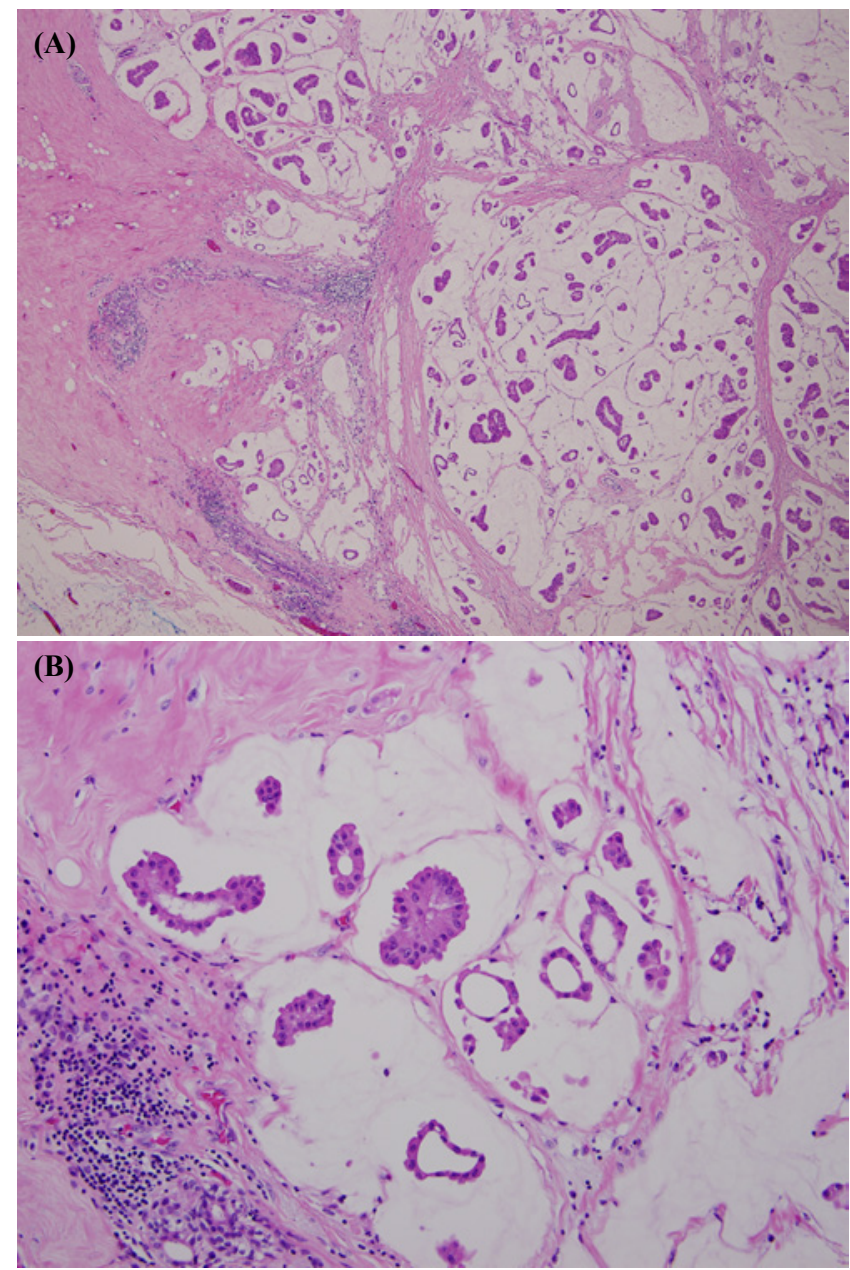

Figure 3: Pathological examination disclosed tumor cells forming micropapillae, pseudoacini and trabeculae, floating in the mucinous pools (a). The micropapillary architecture exhibited a central space and a serrated outer border (b). (H\&E; a, $x 40 ; b, x 200)$.

cytology. Cytologic features such as cellularity, shape of the epithelial cell nests, nuclear pattern, background and stromal component are helpful in the differential diagnosis $[1,5]$. The mucinous material in mucinous carcinoma appears thin and wispy or thick and resembling colloid on aspiration biopsy smears. In general, the cytologic pattern is highly variable from predominantly dyscohesive single epithelial cells floating in a mucinous background to predominantly cohesive sheets and three-dimensional aggregates. Cellular atypia is mild to moderate. A distinct feature of mucinous carcinoma is the presence of thin-walled capillaries, either free-floating or coursing through the thick mucin [8]. Caution must be taken in diagnosing any malignant mucinous lesion with a high nuclear grade specifically as mucinous carcinoma, because these lesions most likely will harbor ductal carcinoma, NOS component. It is recommended that paucicellular lesions lacking cytologic atypia, whether representative of FCC or MLL, be considered for conservative surgical excision based on the lack of reliable malignant features $[7,8]$.

Invasive micropapillary carcinoma is a morphologic variant of carcinoma that has gained increasing attention in the past decade. It has been recognized as a morphologically distinctive form of ductal carcinoma in which the tumor cells are arranged in hollow aggregates or morule-like clusters. Invasive micropapillary carcinoma is characterized by artifactually created spaces and reverse cellular 
Citation: Liu CY, Liu CS, Wu YY (2011) Cytologic Findings of Breast Mucinous Carcinoma with Micropapillary Pattern: Report of A Case and Literature Review. J Cytol Histol 2:114. doi:10.4172/2157-7099.1000114

Page 3 of 3

polarity [9]. The angioinvasive behavior of micropapillary carcinoma has been recognized. Kuroda et al. [10] reported a significantly less favorable prognosis for micropapillary carcinoma when compared to non-micropapillary carcinoma [10]. When compared to patients with non-micropapillary invasive carcinoma, patients with invasive micropapillary carcinoma have a significantly shorter disease free and overall survival. However, when stratified for the number of lymph nodes and other prognostic factors in multivariate analysis, patients with invasive micropapillary carcinoma have survival rates similar to those with non-micropapillary invasive carcinoma [4].

In recent years, a micropapillary form of mucinous carcinoma has been described. Wai-Kuen $\mathrm{Ng}$, in a review of 556 ductal carcinomas, found five mucinous carcinomas with a diffuse micropapillary arrangement [2]. The author presented them as a new subtype of mucinous carcinoma, morphologically equivalent to a mucinous counterpart of micropapillary carcinoma. In spite of the rare incidence (less than $1 \%$ of ductal carcinoma), the existence of micropapillary pattern in mucinous carcinoma warrants special attention $[2,3,6]$. There were few debates on the contribution of micropapillary pattern toward aggressiveness [11-13]. When compared with invasive micropapillary carcinoma, the clinical course of micropapillary variant of mucinous carcinoma appeared relatively indolent. In a recent study, Ranade et al. [3] evaluated 100 cases of mucinous breast carcinoma, and the emphasized the impact of micropapillary pattern [3]. The incidence of lymph node positivity in micropapillary mucinous carcinoma was higher than lymph node positivity in pure mucinous carcinoma. A micropapillary pattern was identified in $60 \%$ of lymph node-positive pure mucinous carcinomas. The authors concluded that pure mucinous carcinomas with a micropapillary pattern were more frequently associated with nodal disease. However, it remains unclear whether the micropapillary architecture represents the mucinous counterpart of invasive micropapillary carcinoma or a genuine micropapillary variant of mucinous carcinoma.

The implication of micropapillary pattern has been noticed in recent studies for breast mucinous carcinoma. Micropapillary carcinoma can be identified by fine needle aspiration cytology, while micropapillary pattern in mucinous carcinoma is apparently under-recognized. Because of the association between micropapillary architecture and nodal metastasis, the characteristic cytologic and histologic features must be distinguished in a preoperative fine needle aspirate specimen.

\section{References}

1. Gray W, Kocjan G (2010) Diagnostic cytopathology. $3^{\text {rd }}$ ed. Churchill Livingstone.

2. Ng WK (2002) Fine-needle aspiration cytology findings of an uncommon micropapillary variant of pure mucinous carcinoma of the breast: review of patients over an 8-year period. Cancer 96: 280-288.

3. Ranade A, Batra R, Sandhu G, Chitale RA, Balderacchi J (2010) Clinicopathological evaluation of 100 cases of mucinous carcinoma of breast with emphasis on axillary staging and special reference to a micropapillary pattern. J Clin Pathol 63: 1043-1047.

4. Rosen PP (2009) Rosen's breast pathology. $3^{\text {rd }}$ ed. Lippincott Williams \& Wilkins.

5. Cibas ES, Ducatman BS (2009) Cytology: diagnostic principles and clinical correlates. $3^{\text {rd }}$ ed. Sauders Elsevier 2009: 241-243.

6. Madur B, Shet T, Chinoy R (2007) Cytologic findings in infiltrating micropapillary carcinoma and mucinous carcinomas with micropapillary pattern. Acta Cyto 51: 25-32.

7. Sohn JH, Kim LS, Chae SW, Shin HS (2001) Fine needle aspiration cytologic findings of breast mucinous neoplasms: differential diagnosis between mucocelelike tumor and mucinous carcinoma. Acta Cytol 45: 723-729.

8. Ventura K, Cangiarella J, Lee I, Moreira A, Waisman J, et al. (2003) Aspiration biopsy of mammary lesions with abundant extracellular mucinous material. Review of 43 cases with surgical follow-up. Am J Clin Pathol 120: 194-202.

9. Tavassoli FA, Devilee P (2003) World Health Organization Classification of tumors: Pathology and genetics of tumors of the breast and female genital organs. Lyon: IARC Press.

10. Kuroda H, Sakamoto G, Ohnisi K, Itoyama S (2004) Clinical and pathologic features of invasive micropapillary carcinoma. Breast cancer 11: 169-174.

11. Walsh MM, Bleiweiss IJ (2001) Invasive micropapillary carcinoma of the breast eighty cases of an underrecognized entity. Hum Pathol 32: 583-589.

12. Bal A, Joshi K, Sharma SC, Das A, Verma A, et al. (2008) Prognostic significance of micropapillary pattern in pure mucinous carcinoma of the breast. Int J Surg Pathol 16: 251-256.

13. Shet T, Chinoy R (2008) Presence of a micropapillary pattern in mucinous carcinomas of the breast and its impact on the clinical behavior. Breast $\mathrm{J} 14$ 412-420. 